\title{
Note sur l'interconnexion des chutes des Alpes
}

Dans une conférence faite le 20 avril 1927 à la Sociélé des Ingénieurs civils, M. Maroger a montré les magnifique's résultats qu'une organisation rationnelle d'interconnexion entre producteurs et consommateurs d'énergic esl capable de produire.

Dans une région qui était considérée après la guerre comme vouée à une déchéance certaine au point de vue industriel, lorganisme créé par l'Ĺnion des Producteurs d'Electricilé des Pyrénées-Orientales, a contribué dans une large mesure au prodigieux développennent de lindustrie électrochimique el bectrométallurgique, de la distribution de la force el de la lumière et de lélectrification des chemins de fer qui s'y révèle aujourd'hui avec, comme conséquence, le développement de l'aménagement des chutes d'eau.

Celte organisation, dit M. Maroger, créée par une demi-douzaine de chefs dindustrie, acceptant une discipline librement, mais fidèlement consentie, fait vivre dans une parfaite entente leurs industries respectives dont ils ont pu ainsi assurer le plein épanouissement el poursuivre le développement. Son principe est la mise en commun de toutes les disponibilités sur un réceau de transport collectif de manière à en tirer, par leur groupement, la meilleure utilisation possible.

A ce jour, cette mise en commun porte sur une puissance intallée de $267.000 \mathrm{KW}$ pouvant produire annuellement $900 \mathrm{mil}-$ lions de KIVH. En 1926, l'utilisation a été de $86 \%$ du disponible y compris la période de trois mois de hautes eaux et de $95 \%$ en mettant à part ces trois mois. Des liaisons prochaines avec des bassins complémentaires (Massif Central, Rouergue), conduiront à l'utilisation complète.

Une organisation aussi féconde peut-elle ètre envisagéc dans la région des Alpes ?

La situation est ici bien différente de celle des Pyrénées, région depuis peu ouverte à l'industrie des forces hydrauliques et dans laquelle quelques groupes seulement, en voie d'organisation, ont vu leur avenir sérieusement compromis après la crise de 1921 .

Ici, au contraire, un nombre considérablo de Sociétés en présence qui, depuis 30 ans, se sont créées, lant dans le domaine de la production que dans celui de la distribution, de véritables fiefs dans lesquelles elles vivent en état de prospérilé dans un splendide isolement.

l'our alimenter ces domaines, dont les besoins s'accroissaient sans cesse, leurs exploitants, après avoir épuisé les sources d'énergie locales, sont allé chercher d'autres sources d'énergic au loin, parfois jusqu'à près de $200 \mathrm{~km}$. de distance el les lignes d'adduction qu'ils ont ainsi créées ont été faites pour leurs seuls besoins.

Cependant, en ces dernières années, une entente est inlervenue, sous la dénominalion de Sociélé de Lran'port d'énergic des Alpe: entre quelques exploitants de la région lyonnaise el de la région. de Saint-Etienne en vue de la construction el de l'expluitation en commun d'une ligne de 150.000 volts pour l'adduction des forces hydrauliques de la région de la Haute-Isère. Une entente est également intervenue entre un exploitant et une Sociélé métallurgique de la région de Saint-Etienne pour la construction et l'exploitalion en commun d'une centrale sur l'Isère el d'une ligne de transport à 150.000 volts. Mais ce sont la des exemples isolés qui ne groupent d'ailleurs que des consommateurs d'énergie al non des producteurs.

A notre commissance ancune lentative de groupement complet n’a éle faite jusqu’à ce jour parce que l'on pose en principe qu'aucune lentative de celle nature n'a chance de sucés en présence de la diversité, de la puissance, de l'espril d'autorité el d'individualisme des intérêts en présence. On dit aussi que le puissant stimulant de l'électrification des chemins de fer, qui a si grandement facilité l'organisation pyrénéenne, fail défaut dans les $A$ pes et que, par conséquent, l'intégration des consommateurs est aussi difficile à réaliser que celle des producteurs. On dil enfin que dans la multitude de lignes, construites pour des besoinc individuels, qui sillonnent le bassin du Rhône ou dont on a prévu la construction, bien peu pourront servir utilement dans le réseau qu'il y aurail lieu d'élablir en vue d'une intercomnexion rationnelle et économique et que tout est à faire, par conséquent, dans. l'organisation des transports.

Toutes ces objections sont évidemment impressionnantes mais aucune cependant ne nous semble péremptoire.

Il est impossible que lesprit d'individualisme, mème forlifie par une situation puissante, ne cède pas devant un intèrèt individuel certain, doublé de l'intérèt public. Il est démontré que lentente pyrénéemne, qui a laissé à chaque adhérent sa pleine indépendance commerciale et financière, lui a assuré le plein épanouissement et le régulier développement de son industrie.

En second lieu, si, comme dans les l'yrénées, l'électrification des chemins de fer ne se trouve pas à la base de l'organisation dont elle a été un puissant stimulant, on peut affirmer que l'organisation des Alpes sera un puissant stimulant de l'électrification des chemins de fer P.-L.-M. el que colle-ci, facilitée par des alimentations multiples el puissantes, ne lardera pas à se développer el à contribuer au succès de l'entente.

L'objection la plus grave est celle de l'absence de réeaux. Ia solution est simple; elle a été donnée dès 1920 dans le plan d'électrification générale de la France élaboré par le Ministère des Travaux Publics. Sur une grande artère principale, IyonSaint-Ftienne-Avignon-1larseille, viendrait se déverser, par des Lransversales, l'énergie des chules alpestres, elles-mêmes reliées entre elles par des réseaux secondaires. Nlimentéc à l'ouest par le Mass if Central et les Pyrénées, celle artere printipale se poursuivrail au nord jusqu'à Paris par Le Creusol ou I ijon el Auxerre. La solution est simple mais, même limilée à la région des Alpes, elle représente une dépense considérable. Seul un grand organisme spécial, quelque chose comme une (Co l’.-L.-.Il. d'électricité, pourrait entreprendre ce travail aver le concours de l'ilal.

I. monent ne serail-il pas venu pour le's grands producteurs d'énergie, les grands divlributeurs et les grands consommateurs de prendre l'iniliative d'une lelle ceuve et de la réaliser avec l'aide des prestations en nature? Ainsi la région des $\Lambda$ lpes bénéficicrait à son tour des bienfaits d'une organisation qui vient de faire ses preuves d.ms la region pyrénéenne.

I. Dusaugey. 